Review Article

\title{
An Overview of Distributed Energy-Efficient Topology Control for Wireless Ad Hoc Networks
}

\author{
Mohammadjavad Abbasi, Muhammad Shafie Bin Abd Latiff, and Hassan Chizari \\ Department of Computer Science, Faculty of Computing, Universiti Teknologi Malaysia (UTM), 81310 Skudai, Johor, Malaysia
}

Correspondence should be addressed to Mohammadjavad Abbasi; mj_abbasi55@yahoo.com

Received 29 July 2013; Accepted 20 October 2013

Academic Editor: Hongli Dong

Copyright (C) 2013 Mohammadjavad Abbasi et al. This is an open access article distributed under the Creative Commons Attribution License, which permits unrestricted use, distribution, and reproduction in any medium, provided the original work is properly cited.

\begin{abstract}
A wireless ad hoc network is composed of several tiny and inexpensive device such as wireless sensor networks (WSNs) which have limited energy. In this network energy, efficiency is one of the most crucial requirements. Data transmitting in minimum power level is one way of maximizing energy efficiency. Thus, transmission power level of nodes should be managed in a smart way to improve energy efficiency. Topology control is one of the main algorithms used in a wireless network to decrease transmission power level while preserving network connectivity. Topology control could improve energy efficiency by reasonably tuning the transmission power level while preserving network connectivity in order to increase network capacity and lifetime. In pursuit of energy efficiency and connectivity, nodes can be selfish and are conflicting with each other. Therefore to overcome the conflict, game theory is used to construct energy efficient topology, as well as minimizing energy consumption. In this paper, the main goal and most recent energy efficient topology control algorithms in WSNs and ad hoc network are classified and studied according to their specific goals.
\end{abstract}

\section{Introduction}

Wireless sensor networks (WSNs) are a particular type of ad hoc network, in which the nodes are autonomous. These nodes are tiny devices equipped with communication component, data computation, and sensing capability [1-3]. In this type of network, each node collects information from the target area and sends this information to a sink, through a multihop communication network. A wireless network consists of hundreds to thousands nodes, which are deployed either inside the target area or very close to the target area. Upon an event happening or during monitoring sessions, the wireless nodes will collect and report this information to the sink node for further analysis.

These wireless networks can be used for many important applications such as health care, intrusion detection and plants control, weather monitoring, security and tactical surveillance, disaster monitoring, and ambient conditions detection $[1,4]$. As an example, in forest fire early detection system [5], wireless temperature and smoke wireless nodes are installed in the forest to detect fire or smoke in its early stage, without deploying complicated wired structures. Another application is in a battlefield, a soldier can be aware of the status of friendly troops or the availability of equipment by their information collected from wireless networks [6].

Energy efficiency is one of the main requirements in sensor networks [7]. Nodes in wireless sensor network are powered with limited energy resource for variety of applications and thus have limited lifetime. Therefore, the energy resource of sensor nodes must be managed efficiently to improve the network lifetime. Moreover, sensor nodes are equipped with storage device, communication radio, and computation device, all of which are powered with limited battery provision. Thus, it is mandatory that every node be energy efficient; this not only maximizes the node's lifetime but also maximizes the network performance. Designing an energy efficient algorithm for the desirable network performance is necessary.

Wireless networks performance can be enhanced by designing energy efficient algorithm. One way for maximizing energy efficiency is transmitting data with minimum power level. Topology control (TC), that is, the study of how 
to adjust each node power level so as to increase network goals, is an algorithm used to improve network performance. In topology control, sensor network is abstracted as graph consisting of sets of wireless nodes and communication links between these nodes. The role of a topology control algorithm is to dynamically adjust transmission power level of defined set of neighbor nodes for each node. The aim is to construct efficient networks and satisfy energy efficiency, energy balancing, and network connectivity. By minimizing transmission power level, nodes collaboratively set their optimal transmission range instead of maximum transmission level and TC helps constructing energy efficient topology, thereby improving network lifetime. The most important challenges in topology control are briefly summarized below.

(1) Fully Distributed Control. In many scenarios, nodes, which execute a task selfishly to save energy, are expected to work unattended in remote geographic areas under critical environmental conditions. Therefore, topology control method should adopt a fully distributed control structure $[8,9]$.

(2) Transmission Power Selection. Deploying several hundreds to thousands of inaccessible and unattended wireless nodes, which are prone to failures, makes topology control as an ambitious task. Each node selects its own power range while preserving network connectivity by topology control [10-12]. This act must not intervene the formal service in the wireless networks and the system must be enough node degree to increase the network real-time packet delivery and adaptive to select transmission power range.

(3) Energy Balance Topology Control. In wireless network, each node chooses nodes with higher residual energy to minimize the energy consumption of nodes with low residual energy, even though more power is needed [13-15]. Hence, the constructed topology must have the properties such as balanced energy consumption.

(4) Energy Efficient Topology Control. Despite the capacity of battery, wireless networks should operate for a relatively long period of time, since it might be impossible to recharge or replace node batteries. The lifetime can be improved by utilizing the energy-efficient topology control algorithms [16-18].

From the above discussion, clearly good research is necessary to address topology control problem. Hence, during topology control, it is desired to obtain the minimum transmission power levels of the nodes while preserving the network connectivity capability. Although, the minimum transmission power levels of network is important during topology process, the energy efficiency and energy balance across the topology control should be maintained during the operation.

In wireless network, nodes act selfishly and conflict with each other in pursuit for energy efficiency and connectivity [19-21]. If the nodes select lower transmission range, the constructed topology will be disconnected. In reverse, if nodes select high transmission power level, the interference among nodes will raise, leading to high energy consumption. The main problem is how to establish a tradeoff between connectivity and energy efficiency for each node. Game theory is used to solve the conflicting objectives of nodes seeking to achieve connectivity and energy efficiency.

The rest of this paper discusses energy efficiency topology control issues in WSN and highlights the limitations of existing topology control algorithms. After describing the basics of ad hoc and wireless sensor networks in the following section with some examples for each category of the detailed topology control taxonomy presented in Figure 1, game theory is briefly elaborated in Section 2. Section 3 discusses heterogeneous transmission power. The homogeneous transmission power control is discussed in Section 4, which consists of centralized and distributed energy efficient topology control algorithms. Topology control algorithms are discussed in Section 5. Finally, Section 6 discussed conclusions.

\section{Game Theoretic Definition and Preliminaries}

Game theory is one of the fundamental mathematical tools that has been used for analyzing between rational and intelligent players. Game theory has been used in a system, with regard to action and pay-off. A review of some fundamental definitions and concepts in game theory that will be used and applied throughout this research are stated in the literature $[22,23]$.

2.1. Game, Strategy, and Equilibrium. A game consisted of players, the possible strategy of the players, and consequences of the strategy. The definition of the game is given in Definition 1.

Definition 1. A game $\Gamma$ has three elements $\langle I, A, u\rangle$, where

(i) player set $I=1, \ldots, n$, where $n$ is number of players in game,

(ii) action set $A=\times_{i \in I} A_{i}$ is the space of all action vector, in which each $a_{i}$ of the vector $a \in A$ belongs to the set $A_{i}$, the set of actions of player. It is the Cartesian product of an action for each player $i$,

(iii) $u$ is a utility of player $i$ over outcomes defined by strategy profile. For a particular action $a, u(a)=$ $\left(u_{i}(a), u_{2}(a), \ldots, u_{i}(a)\right)$ is called a individual utility function $u_{i}(a)$.

In wireless network infrastructure, the nodes are often the players during a game, in which the nodes are constructing a connected topology. Recall that some of the features under its control are classified by transmission power (action list) $p$. Thus, the best action might be chosen from the transmission power list $p$. In essence, the collection of the best actions determines the outcome of the game. Transmission power level considers the outcome with network connectivity by minimum energy consumption. However, some players are conflicting with each other by this outcome. Game theory 


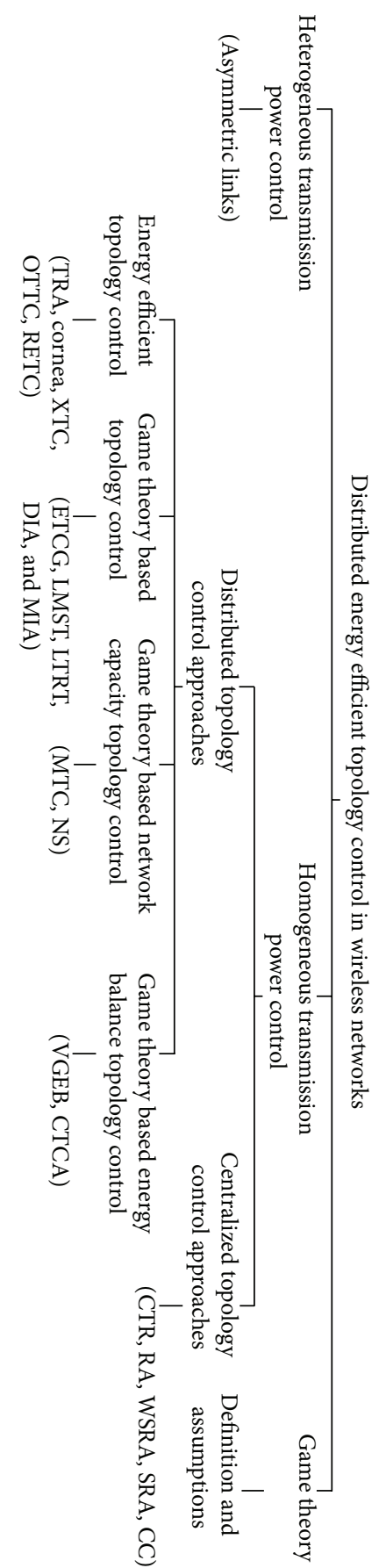

FIgURE 1: Taxonomy of energy-efficient topology construction protocols.

can predict this outcome among the game. Nash equilibrium $(\mathrm{NE})$ is one of the best solutions for outcome problem in game theory $[24,25]$. An NE has mean features and is a fixed point. From this fixed point, no player has any incentive to deviate from its action. Therefore, an NE can predict the outcome of a game.

Definition 2. The action profile $a^{*}$ is an NE, if $u_{i}\left(a^{*}\right) \geq$ $u_{i}\left(a_{i}, a_{-i}^{*}\right)$ for every action $a_{i}$ of a player $i$.
Based on Definition 2, the most action of a player is to avoid unstable strategy and play in the best strategy. When many strategies exist, it denotes a best response for each player, given the strategies of the other players. So that player can pick its best response accordingly.

Definition 3. An action $a^{\prime} \in A_{i}$ is the best response of strategy for a player $i$ if and only if $u_{i}\left(a_{i}^{\prime}, a_{-i}\right) \geq u_{i}\left(a_{i}, a_{-i}\right)$, for all $a_{i} \in$ $A_{i} \backslash a_{i}^{\prime}$. 
Definition 3 elaborates why NE is the stable point of the best response. Based on existence of pure strategy of NE, stable point of NE can be found. Sometimes game has multiple equilibria. The task of getting rid of the undesirable ones is another issue which needs more attention. Furthermore, one player wants the convergence of the game properties and the problem is further compounded when some NE exists.

There is a special class of game theory for existence of the best strategy and convergence to the properties. Denote how Definition 3 can help for best strategy-based algorithm to come up with some NE of the game.

2.2. Potential Games. In a potential game, the consequences of any individual player's change in strategy are implied by the potential function. General games do not need to possess pure Nash equilibrium, while this is not the case for potential games [26]. Beyond merely possessing pure Nash equilibrium, potential games also provide a straightforward algorithm for agents to learn to play a pure Nash equilibrium and the best response dynamics. Moreover, the best response dynamic is the simplest of the learning dynamics.

Definition 4. Ordinal potential function (OPF): a game is an ordinal potential function $\Phi: A \rightarrow R$ such that for any player $i$, any joint action $a \in A$ and any action $a \in A_{a}$ :

$$
\begin{aligned}
& u_{i}(a)-u_{i}\left(a_{i}, a_{-i}\right) \\
& =\phi_{i}(a)-\phi_{i}\left(a_{i}, a_{-i}\right) .
\end{aligned}
$$

$\phi$ is Exact Potential Function (EPF).

Definition 5. A game $\Gamma$ is an ordinal potential game (OPG) $a \in A$ and any action $a \in A_{i}$ :

$$
\begin{aligned}
u_{i}(a)-u_{i}\left(a_{i}, a_{-i}\right)>0 \\
\quad \Longleftrightarrow \phi_{i}(a)-\phi_{i}\left(a_{i}, a_{-i}\right)>0 .
\end{aligned}
$$

According to Definitions 4 and 5, an EPG is an OPG with the similar potential function. Potential games with action vector are known to be fixed at least on one $\mathrm{NE}$ as the best strategies [26]. The following lemma shows how NE of the game can be classified.

Lemma 6. Let $\Gamma$ be an OPG and let $\phi$ be its corresponding OPF. If $a \in A$ maximizes $\phi$, then it is a Nash equilibrium.

Proof. From Definition 5 we have that $\Gamma$ is an OPG. $\Gamma$ is better response dynamic and has defined an improvement path, a sequential of improving action, which is finite. The equilibrium set of $\Gamma(u)$ coincides with equilibrium set of $\Gamma(\phi)$. Then, $a \in A$ is an equilibrium for $\Gamma$ if and only if for every $i \in I, \phi(a) \geq \phi\left(a_{i}, a_{-i}\right)$ for all $a_{-i} \in A_{i}$. Consequently, if $\phi$ used a maximal point in $A$, based on the finite improvement path (FIP) property of potential game [26], $\Gamma$ can be converge to equilibrium. And each player can maximize its utility, as far as possible to maximize potential function. Based on Nash equilibrium, no node can maximize its utility by changing its action. $\Gamma$ can converge to the Nash equilibrium.
Based on Lemma 6, potential function is a subset of the $\mathrm{NE}$ of a potential game. For identification of NE in game, it is required to identify the potential functions. In addition to its $\mathrm{NE}$, potential games have convergence properties, yet, selfish adaptations. The balance between efficiency and stability is fundamental for any dynamic system, specifically in a mutual system of independent selfish players. Moreover, in NE, sense stability is based on self-interest whereas system efficiency is based on general interest. Prisoners' Dilemma in [27] is a classical sample that demonstrates the inefficiency of the stable outcomes. The efficiency concept is called Pareto optimality in game theory.

Definition 7. An action set $a^{\prime}$ is Pareto optimality (OP) $a \notin A$ such that $u_{i}(a)>u_{i}\left(a^{\prime}\right)$ and $u_{j}(a)<u_{j}\left(a^{\prime}\right)$ for $j \in A$.

Definition 7 elaborates how, from a Pareto Optimal (PO) state, it is inconceivable to change into another state and increase the pay-off of agents without minimizing the payoff of some other agents. The terminologies and definitions illustrated in this subsection are used as the preliminaries of review algorithms art of the misbehavior mitigation system. In the rest of the paper, the above terminologies and definitions will be used frequently.

\section{Heterogeneous Transmission Power Control}

In heterogeneous networks, nodes have different transmission power levels. The assumption of heterogeneous nodes does not hold the same type; therefore, they may have slightly different maximal transmission power. There also exist heterogeneous wireless networks in which devices have dramatically same capabilities. TC using per node transmission power method has been proven to be more efficient in improving the network lifetime. Asymmetric link algorithm which is used in heterogeneous nodes to efficiently adjust a power level had been proposed in [28]. Moreover, each node has different maximum transmission levels since they are heterogeneous. Furthermore, for node $i$, the current transmission power is $p_{i}, P_{i j}$ as the transmission power required for node $i$ to connect a node $j$, and $P_{i}(\max )$ as its maximum transmission power level. Since $P_{i}(\max ) \neg P_{j}(\max )$ for $i \neg j$. In such a situation where $P_{i}(\max ) \geq P_{i j}>P_{j}(\max )$, there exists a reverse link in the connected topology since $P_{j i}>P_{j}(\max )$. Consequently, the introduced algorithm seems to be stable. Each node based on its information adjusts the transmission powers. Thus, the topology converges to the final topology with minimum transmission power. However, the notion of the most existing topology control algorithms cannot be directly extended to heterogeneous networks where different nodes may have various transmission power level.

\section{Homogeneous Transmission Power Control}

In homogeneous topology control algorithms, the connected topology is constructed by adjusting the transmission power level of the nodes. In the first case considered in this section, all the nodes are homogeneous infrastructure in a network. 
For this case, coverage, connectivity, and energy efficiency algorithms are presented.

The algorithm in the branch of transmission power control are more coverage oriented $[29,30]$. However, in such cases, connectivity is not preserved. Moreover, the coverage oriented protocols used the different techniques for construction of topology control. Some algorithms are introduced to provide coverage of a set of predefined target areas. Furthermore, the algorithms in the branch transmission power control are focused only on construction of a connected network. The main aim of these algorithms is to decrease transmission power of the topology, while preserving connectivity.

The proposed algorithm in [31] used disk model algorithm and studied link connectivity. In summary, the disk model is Boolean: an edge $(i, j) \exists$ for all $i$ \& $j$ only if power level $p_{i}>w(i, j)$, otherwise not. Energy efficiency is a key requirement in the design of wireless networks, since nodes have limited operational life. Hence, energy-efficient algorithms are required to adjust communication links between nodes. On the other hand, it has been shown that the topology of a sensor network greatly affects the nodes operational life [32].

Topology of each network depends on controlled and uncontrolled factors. Controlled factors are transmission power level and use of directional antennas. Uncontrolled factors are classified as node failure, node mobility, noise, and interference [46]. This section discusses controlled factors in wireless networks with focus on transmission power selection algorithms. The efficient topology control has significant effect on network capacity and network operation lifetime. Many TC algorithms have been introduced for homogeneous networks. Such algorithms deal with limitations of wireless networks, such as energy consumption and network capacity. Such proposed algorithms are analyzed critically. Comparisons of the energy efficient topology control algorithms are also given in Table 1. In the following subsection, centralized and distributed topology control algorithms are discussed.

\subsection{Centralized Topology Control Algorithms. Centralized} transmission power control method is stable topology construction algorithm. There are many algorithms from the topology control that can be applied as centralized transmission power control method. However, in centralize topology control, node needs the authority to control its power levels.

Authors in [47] applied minimum spanning tree (MST) on a graph that preserves connectivity. Furthermore, the transmission power level of each node is guaranteed as long as the greatest link of the MST is established and this is referred to as connected topology. In [47], authors investigated that for connected networks, the weight of the greatest link, from which the level of the critical transmission range (CTR) can be computed, is represented as being with high probability $(w: h: p)$ by

$$
\mathrm{CTR}_{\text {dense }}=\sqrt{\frac{\log n+f(n)}{n \pi}},
$$

where $f(n)$ is a nondecreasing function of $n$, such that $\lim _{n \rightarrow \infty} f(n)=+\infty$, and $\log n$ is the logarithm of $n(\log n)$.

However, $(w: h: p)$ of (3) works only for 2-D deployments. Authors in [3], used similar equation which works for 1-D and 3-D deployments. Moreover, $(w: h: p)$ has several limitations. It just can be used for connected networks and it is not precise.

Authors in [38] reformulated the CTR methods, which can preserve network connectivity in both sparse and dense graph. Moreover, it uses the size of the deployment area and computes the optimum radio range and number of nodes to establish a connectivity. For the one-dimensional case, the CTR is given by

$$
\mathrm{CTR}=k \frac{l \log l}{n},
$$

where the value of $k$ is a constant with $1 \geq k \geq 2$, and $l$ is the size of the area. Moreover, the researchers also provided a partially proven objective for $d$-dimensional deployments.

For $d$-dimensional deployments, with $d=2 ; 3 ; \ldots$, the authors in [3] introduced a partially demonstrated result to find the CTR for preserve connectivity as

$$
\mathrm{CTR}=k \frac{l^{d} \log l}{n},
$$

where $k$ is a constant with $0 \leq k \leq 2^{d} d^{d /(2+1)}$.

The CTR is generally a hard and costly operation, particularly when a full connection is required. Moreover, the CTR may be near to the maximum transmission power level and therefore there will be neither differences in the topology nor energy efficiency.

Authors in [48] proposed algorithms to find a solution for range assignment (RA) problem. The algorithms find a strongly connected graph and minimize the total energy usage of the network. An approach is proposed in $[48,49]$ for solving the RA problem in 2-D and 3-D. Furthermore, symmetry constraints have been added to the RA problem. Moreover, adding symmetry constraints has resulted in two new issues: first, weakly symmetric range assignment (WSRA) and second the symmetric range assignment (SRA). The solution to the SRA problem is required as all edges in the construction topology should be a reverse link. It has been assumed that some nodes have to maximize their power level to build the symmetric tree. On the other hand, the WSRA removes directed links and adjusts the RA for each node such that the comprised topology is connected and symmetric. The total energy usage of all the assignments is minimized. The WSRA method is more flexible to solve transmission range assignment because it constructs a connected backbone of reverse links. Furthermore, WSRA had been proposed in [3] which has a great gain compared to the SRA in terms of energy consumption.

The aim of TC algorithm is to specify the level of transmission power in order to ensure connectivity and minimize energy usage for each node. The researchers $[32,36,41]$ proposed some TC algorithms to reduce power level and ensure network connectivity. In [39], authors studied cooperative communication (CC) algorithm which allow multiple nodes 


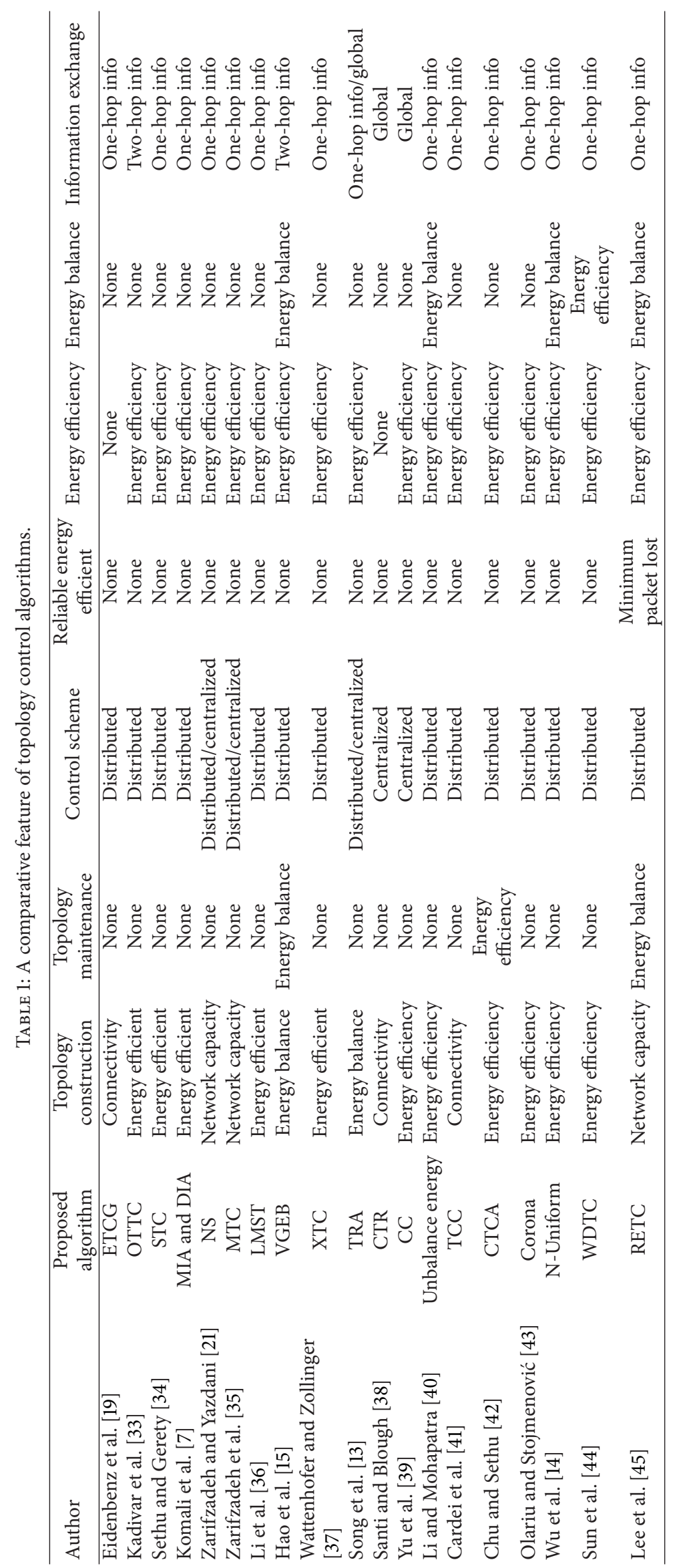


to simultaneously forward the same packet. CC algorithm considers disconnected topology and the improvement of a centralized TC scheme, named Cooperative Bridges. CC minimizes the power levels as well as preserve network connectivity. The $\mathrm{CC}$ algorithm defines such a problem as follows. First, TC is considered as extended links caused by cooperative communication. Second, the energy efficiency link is extended with cooperative communication. The main aims of proposed centralized topology control technique in [39] are reducing the transmission power level of nodes and increasing connectivity for bipartite networks. Accordingly, simulation results show that CC technique has better performance than other techniques in terms of the connectivity to the energy consumption ratio. However, in CC algorithm, each node is responsible for constructing efficient topology based on global information.

4.2. Distributed Topology Control Algorithms. The main concern of the distributed topology construction algorithm is building a "quality" and efficient topology. In such topology, efficiency refers to minimal energy usage, minimum computational and information exchange complexity, and so on. The rest of this section discusses the energy-efficient topology control in wireless networks and highlights the limitations of existing topology control designed to handle energy efficiency in homogeneous networks.

4.2.1. Energy Efficient Topology Control Algorithms. In [50], the authors argue that, for large size of network, there is still amount of battery left unused after the operational life of the sensor network is over. This unused battery can be up to $90 \%$ of total initial energy. To balance the uneven energy consumption among the nodes in wireless networks, mixed routing algorithm (MRA) is proposed by authors in [51]. In MRA algorithm, each node is allowed to either forward a packet to one of its neighbors or to forward it directly to the destination. Furthermore, the decision is depending on its residual energy. The drawback of this algorithm is that it cannot be applied in networks when the node's maximal transmission level is smaller than the network area radius.

Authors in [43] discussed the relationship between the network operational life and the width of each corona $C$ in concentric corona model. The researchers proposed algorithm that minimized the amount of energy usage on forwarding along some intermediate nodes, from a node in a corona and ending at the destination. Moreover, all the Cs in such algorithms must have the same width. However, authors in [43] assumed that all nodes in corona $C_{i}$ should route along a path in $C_{i-1}$, and the power level in $C_{i}$ is $\left(r_{i}-r_{i-1}\right)$ and $r_{i-1}$. Furthermore, if each corona has similar width and similar power level, this assumption may result into the use of less energy for routing. Additionally, divide the corona $C_{i}$ into two subcoronas; namely, $s_{1}$ and $s_{2}$ may lead to consume more energy for packet forwarding. The width of subcorona $s_{1}$ is equivalent to that of corona $C_{i-1}$, so nodes in $s_{1}$ will forward packets to $C_{i-1}$. Furthermore, the nodes in subcorona $s_{2}$ which are near to corona $C_{i-1}$ with power level greater than the width of corona may result in routing across corona
$C_{i-1}$ to corona $C_{i-2}$, that is, more near to the destination. Moreover, as illustrated in [43], the packet forwarded from all nodes in corona $C_{i}$ should be routed for the next intermediate in corona $C_{i-1}$ rather than corona $C_{i-1}$. However, these nodes in $s_{2}$ with power level $\left(r_{i}-r_{i-1}\right)$, which can forward packets to $C_{i-2}$ but should send to $C_{i-1}$, will consume more energy for routing.

In [40], authors studied the problem of unbalance energy consumption in large-scale wireless networks. In such investigation the authors described the energy hole in a ring model. Ring model considers the per node energy consuming rate (ECR) and the per node traffic load. Based on ECR algorithm each node around destination needs to forward more packet as compared to the other nodes which are far away from destination. However, in such algorithm, energy consumption rate is higher in inner rings than outer rings and thus has much shorter operation life.

Authors in [14] proposed a nonuniform node distribution strategy to achieve subbalanced energy consumption based on Corona $C$ models. The research studies show that if the total numbers of nodes in corona grow from $C_{R-1}$ to $C_{1}$ with a common ratio $q>1$ and there are $N_{R-1} /(q-1)$ nodes in $C_{R}$, then the network can improve sub-balanced uneven energy usage. Here, $N_{i}$ represents the entire number of nodes in corona $C_{i}$. However, distribution strategy of nodes cannot work easily, because in most situations, the nodes are distributed randomly.

Corona $(C)$ model with adjustable transmission power with circular multihop deployment is proposed by authors in [13]. The proposed model assumed that the decision factor for optimizing the network operational life minimizes the transmission power of nodes in each $C$. Based on this factor, it divides the maximal transmission level of nodes into several levels. Nodes in the similar Cs have the related transmission level, and different $C$ has various transmission levels, which construct an order list in term of the transmission level order. They used multiobjective optimization problem (MOP) for searching of transmission power level between all Cs. Each node can adjust its transmission power for saving energy. The model divided $t_{x z}$ into $k$ spaces, and sensors have $k$ space transmission power level to choose. The unit length of transmission power range is represented by $d$. Furthermore, the model divides the whole network area with radius range $R$ into $m$ adjacent concentric parts termed $C$ as shown in Figure 2. Authors in [13] also proposed distributed and centralized algorithm for adjusting transmission power of each node in each corona to extend the network lifetime as shown in Figure 3. However, searching the optimal transmission levels of nodes between all the coronas is an NP-complete problem.

The approach in [37] proposed eXtreme topology control (XTC) algorithm for topology control which operates with the neighbors' link qualities. The main features of XTC algorithms are relevant properties (symmetry, connectivity, sparseness, and planarity) of TC while being faster than any previous algorithms. The XTC algorithm does not require the node coordinate information.

The following explanation shows how nodes order list can be established in XTC algorithm. Based on maximum 


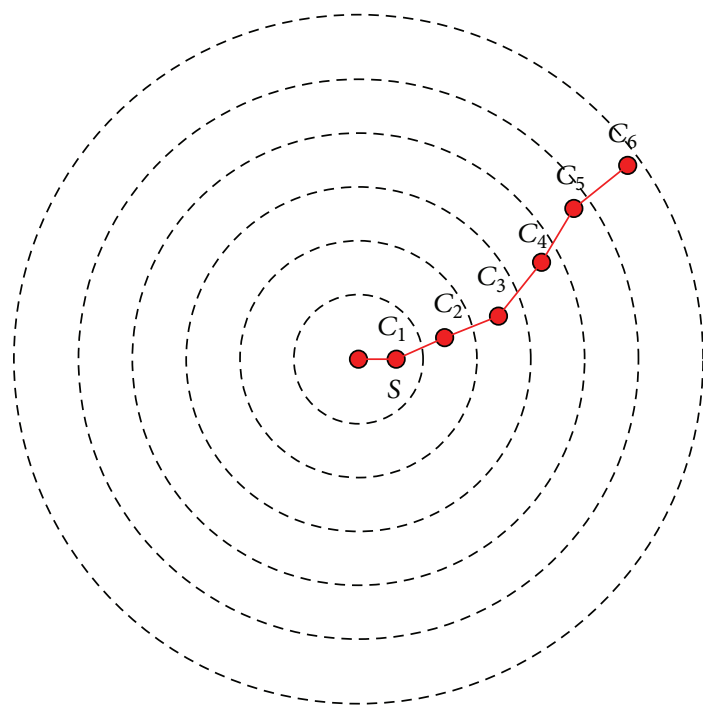

(a)

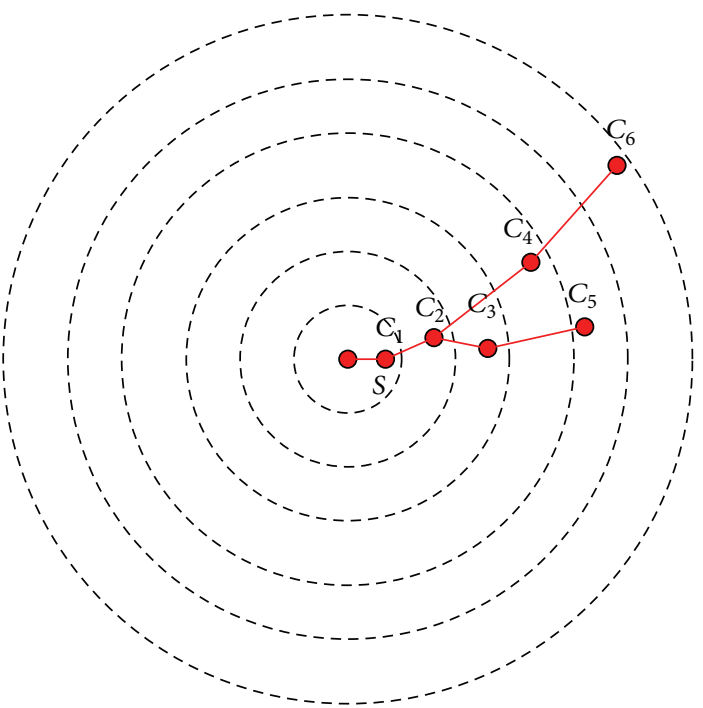

(b)

FIGURE 2: Two branches of transmission powers between all coronas: (a) $k=1$. (b) $k>1$.

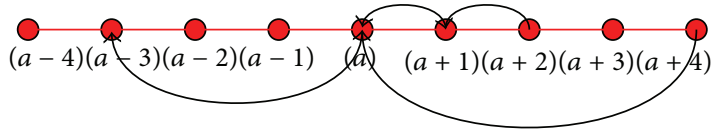

FIgURE 3: Adjacent coronas $k>4$.

transmission power, information of each node can be realized by the weight of the links. With additional assumption, link between nodes is bidirectional. Moreover, the signal threshold can be calculated based on the Euclidean distance to the senders. If all nodes send a message with same transmission power range, the neighbor list of node $u$ is also equivalent to the order list. The link qualities $w_{u v}, w_{u w}$, and $w_{v w}$ reflect that the signal threshold between node $u$ and node $w$ is spoiled by a obstacle, where $w_{u v}<w_{u w}$ and $w_{v w}<w_{u w}$. In contrast, XTC algorithm does not include the link $(u, w)$ in its result topology but applies connection that establishes order list via node $v$. However, the XTC algorithm does not consider the "power stretch mean" and "stretch factors."

One-hop two-hop topology control (OTTC) [33] is an improved algorithm of XTC which operates with weight of the links. Each node collects its one-hop neighborhoods in a neighbor list and exchanges the list between its neighbors. OTTC algorithm supplies connected subgraph with all properties of topology control (i.e., "connectivity," "symmetry," "spanner" and "low degree"). The OTTC works in fully distributed and low quality information. The main issue considered by OTTC algorithm is the "node degree," "stretch factor," and "power stretch mean" factors.

The following description shows how a node's neighbor list can be obtained in the OTTC as shown in Figure 4. Link quality of the node can be realized by having each initial transmission power of each node via hello message. With the additional assumptions, node $u$ can establish its order list by calculating Euclidean distance and signal thresholds. After receiving the order list by each node, nodes $u$ and $v$ exchange their order list. Therefore, after exchanging the neighbor list, node $u$ finds its two-hop neighbor nodes. Node $u$ and node $v$ are connected in the graph, so there exists a path $P: u=u_{0}-$ $u_{1}-u_{2}-\cdot u_{k}=v$ on graph $G$. Edge $u_{j}, u_{j+1} \in P$ with minimum weight $\left\|u_{j}, u_{j+1}\right\|$ between connected link in $G$ which are not connected in the graph $G_{\text {OTTC. Since }} u_{j}$ and $u_{j+1}$ are not in topology $\left(u_{j}, u_{j+1} \notin G_{\text {OTTC }}\right)$, there are two case: First, there exists node $\left.w \in\left(N\left(u_{j}\right)\right) \cup\left(\widetilde{N\left(u_{j}\right)}\right) \cap\left(N\left(u_{j+1}\right)\right) \cup\left(\widetilde{N\left(u_{j+1}\right.}\right)\right)$ such that $w \prec_{u_{j+1}} u_{j}$ and $w \prec_{u_{j}} u_{j+1}$. Since $u_{j} u_{j+1}$ is lower weight, it includes $w \in\left(N\left(u_{j}\right)\right) \cup\left(N\left(u_{j+1}\right)\right)$. Moreover, path $u_{j}, w$, $u_{j+1}$ is connected to $u_{j}$ and $u_{j+1}$. Second, there exists $w \in$ $\left.\left(N\left(u_{j}\right)\right) \cup\left(\widetilde{N\left(u_{j+1}\right.}\right)\right)$ such as $u_{j} u_{j+1}>\max \left\{u_{j} w, u_{j+1} z, w z\right\}$ for some $z$ in second hop $\left(u_{j}, u_{j+1}\right) \cap\left(u_{j, w}\right)$. Furthermore, nodes $u_{j}$ and $u_{j+1}$ are connected with path $u_{j}, w, z, u_{j+1}$.

The proposed OTTC algorithm is fully distributed and work based on low quality information. The main feature of OTTC compared to other algorithms is the use of twohop information exchange between neighbors, which helps to minimize the power level and degree of the nodes. However, OTTC algorithm considers the low degree which reduces the robustness of topology in real-time application. Moreover, OTTC algorithm does not consider the remaining energy and it may result in consuming more energy.

The paper in [44] presents WDTC algorithm which considers residual energy information to the construction of connected topology. WDTC works based on link weight function. Then each node induces minimum spanning tree (MST) in the new weighted graph. At each iteration of T, the link weight is different, so that the induced MST is different as well as initial topology. WDTC algorithm can effectively improve the network operational lifetime and balance the nodes' energy usage.

Distributed and reliable energy-efficient topology control (RETC) algorithm is proposed by authors in [45]. 


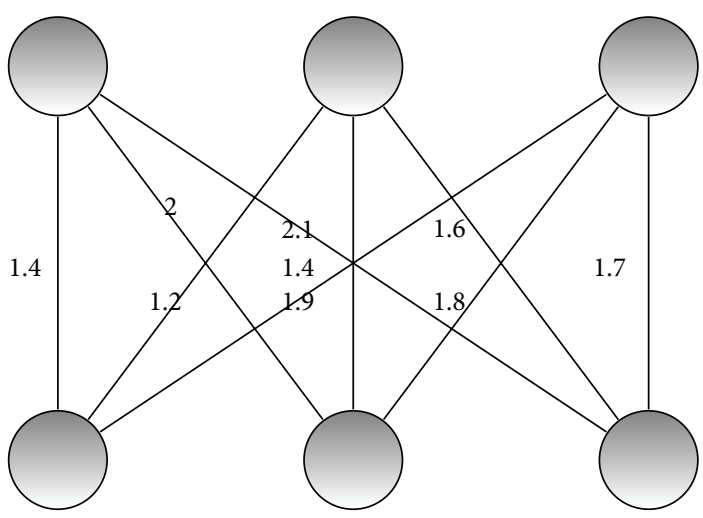

(a)

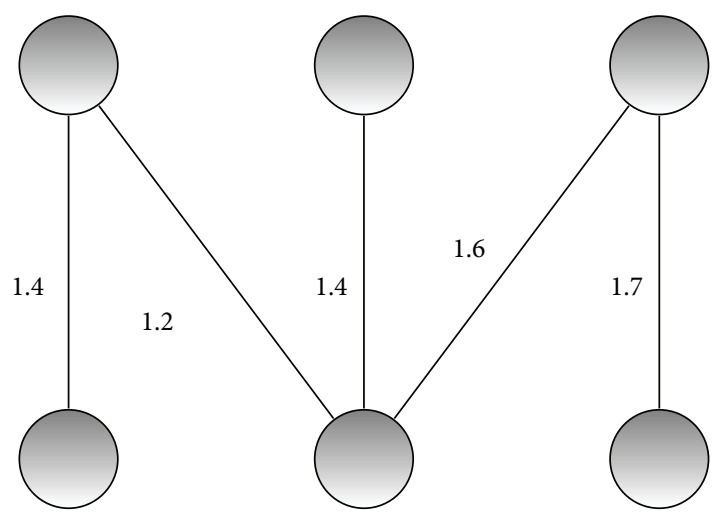

(b)

Figure 4: (a) The original graph $\left(k_{3} ; k_{3}\right)$. (b) OTTC topology control.

The RETC algorithm assumed that nodes are connected to their neighbors with a certain packet loss probability. However, many intermediate nodes and congestion may result in packet loss. Thus, reliability must be achieved while designing topology control. Reliable topology can achieve energy balancing and connectivity. The authors argued that considering only topology control is not sufficient to improve an energy-efficient topology construction algorithm. They used maintenance phase that can balance the energy consumption in order to improve network lifetime. In maintenance phase, topology status and trigger topology construction are monitored by nodes when necessary to maximize network lifetime. Additionally, in RETC algorithm, nodes enable us to autonomously select reliable link which has high probability of packet forwarding. Therefore, in the new proposed topology control, a reliable topology is generated to maximize network reachable probability. However, RETC algorithm only considers the nodes residual energy and does not consider average energy of nodes, which may result in consuming more energy.

4.2.2. Game Theory Based Topology Control Algorithms. In [19], authors were the foremost to propose the equilibrium in topology control Game (ETCG) and studied strong connectivity properties. However, in the proposed algorithm, the stable point of $\mathrm{NE}$ is not guaranteed and also does not consider the energy efficiency. However, the work was assigned to the analysis of complexity in finding an NE.

The approach in $[52,53]$ used the game-theoretic concepts which is "mechanism design" to solve TC problem. Those algorithms are considered to develop globally energy efficient algorithms. Moreover, proposed algorithms are used for designing incentive compatible objective. The aims of those algorithms are global energy efficiency by cooperating the selfish user with the social outputs. "Mechanism design" is applied to provide the appropriate incentives to the individual player. "Mechanism design" can increase their objective function when the topology uses minimum energy consuming, subject to preserving network connectivity. Both authors adopted "mechanism design" algorithm by engineering a payment that leads selfish nodes to forward packets to other nodes. The utility function proposed by author in [53] eliminates that each player realized the per link price that it proposed to pay for forwarding packet. However, the algorithm of assigning price on per edge does not account for the wireless advantage. In addition, the communication cost is increased by a node when transmitting data via an edge as a function of transmission power that is required to obtain the edge. Furthermore, nodes increase uniform energy usage in constructing the edge to each of its accessible neighbor at the same transmission level.

The authors in [9] reformulated the ETCG algorithm as exact potential games (EPG). EPG responded to the existence of at least one NE. EPG responds to the existence of at least one NE. Authors in [7] investigated TC to adjust the per node power level such that the resulting topology was energy efficient and satisfies current global properties such as strong connectivity. The algorithm assumes that nodes are responsible to construct topology. The authors studied Nash equilibrium for constructing efficient topology, when nodes employ the greedy best response algorithm. Based on NE in such methods, a modified algorithm based on a better response dynamic is introduced.

The max-improvement algorithm (MIA) and $\delta$-improvement algorithm (DIA) are proposed by authors in [7]. Both algorithms consist of three main phases: "initialization phase", "adaptation phase," and "update phase." However, such algorithms differ in adaptation phase. In MIA, nodes adjust their transmission power level abased on a "greedy" better response process. On the other hand, in the DIA algorithm, nodes adapt their transmission power range based on "restrained" best response process.

The three phases are discussed as follows.

(i) Initial Phase. Each node $i$ adapts its transmission power to $p_{i}^{\max }$ and discovers its neighbor by sending "hello messages" and then receives the ACKs from each node in the $p_{i}^{\max }$ neighbor. Furthermore, when a node $i$ receives a response ACKs from each transmission power of neighborhood nodes $j$, node $i$ adapts its edge state quality between node $i$ and 


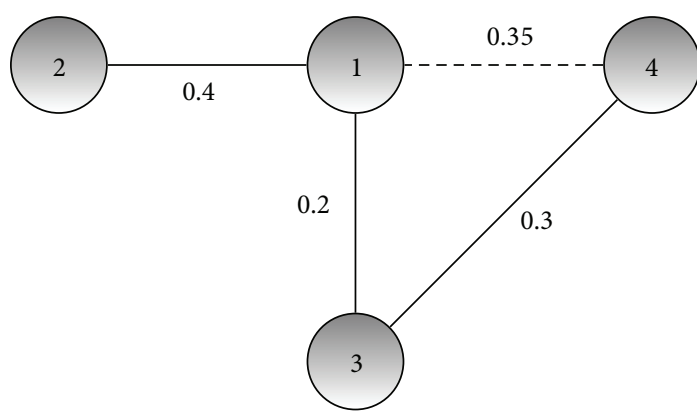

(a)

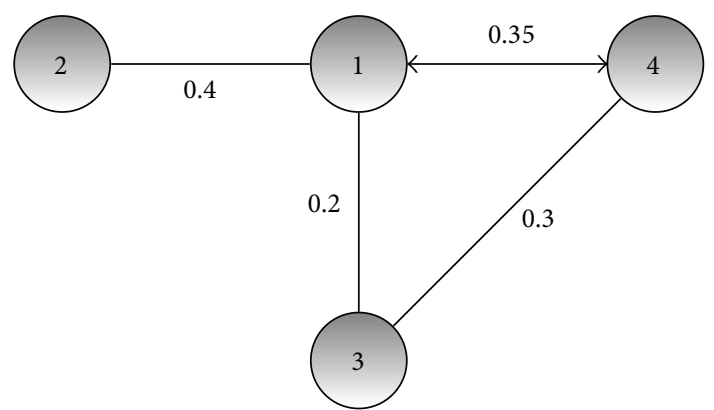

(b)

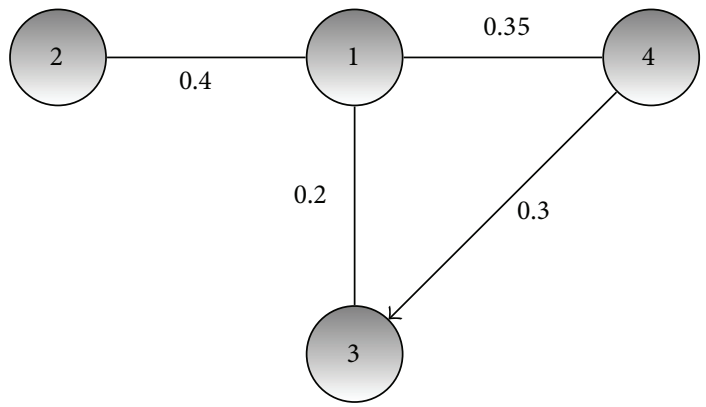

(c)

Figure 5: (a) The MST algorithm. (b) The PMST. (c) The $G_{\text {DIA }}$ constructed by DIA.

node $j,\left(m_{i j}\right)$ to 1 . Intuitively, for each node $i$, determine a link quality $e_{i j}$ as

$$
e_{i j}= \begin{cases}1, & \text { if } p_{i} \geq w(i, j) \\ 0 & \text { otherwise }\end{cases}
$$

Here, $w(i, j)$ is the power level required to reach an edge $i j=$ $(i, j) \in E$. For each node $i \in N$, defines action set as

$$
A=\left\{p_{\max }=p^{0}, p^{1}, \ldots, p^{k}=p_{\min }\right\},
$$

where $A$ is an ordered set (finite number of power levels); that is, $p^{k}<p^{k-1}$. One way to construct $A$ is to let the transmission power level of all nodes be initialized maximum power level and minimize the power level in a step of predefined step size $\delta$.

(ii) Adaption Phase. In adaption phase, each node is selected from permutation round robin to assign its transmit level. All those nodes produce either MIA or DIA during the game and only one node sets its transmission range. Node $i$, selected via some sequential orders, improves its pay-off as shown in (8), by adapting its transmission power from $p_{i}^{\max }$ based on $p_{i}<p_{\text {max }}$ :

$$
u_{i}(p)=\varphi_{i}(g(p))-\chi_{i}\left(p_{i}\right)
$$

where $\varphi_{i}: G \rightarrow I R$ represents the pay-off node $i$ proceed from graph $g$, and $\chi_{i}$ is the cost increased. Both algorithms determine the adaption phase in two categories. In the MIA adaptation phases, the game can be played as a normal game, where every node is chosen to increase its individually utility in that iteration. In the best response based algorithm, whenever a node has a chance to change its action, it chooses an action that increases its individual utility shown in (8), given the transmission power range as shown in Figure 5 of all other nodes based on the following

$$
\begin{gathered}
p_{i}=\underset{q_{i} \in A}{\arg \operatorname{Max}} u_{i}\left(q_{i}, p_{-i}\right), \\
\min _{\widetilde{c}_{i}\left(a_{-i}\right) \in \Re\left(\Gamma_{i}^{c_{i}}\right)} u_{i}\left(\widetilde{a}_{i}^{*}, \widetilde{c}_{i}\left(a_{-i}^{*}\right)\right) \\
\geq \min _{\widetilde{c}_{i}\left(a_{-i}\right) \in \Re\left(\Gamma_{i}^{c_{i}}\right)} u_{i}\left(\widetilde{a}_{i}, \widetilde{c}_{i}\left(a_{-i}^{*}\right)\right) .
\end{gathered}
$$

On the other hand, in the DIA adaptation phases, power is in the discrete action list. More precisely, it is sufficient to search for the best transmission power over that action list which corresponds to the signal's threshold entries of $\Omega$. In DIA algorithm, each node $i$ selects a power level one less than its current power level if the selected transmission power results in a higher utility than its current power level. Otherwise, the node changes transmission power to the power level, that is, currently transmit at. Additionally, given the action of all other nodes, each node chooses to forward a packet to the next node, at power level given

$$
\widetilde{p}_{i}=\underset{q_{i} \in\left\{p_{i}^{k+1}, p_{i}^{k}\right\}}{\arg } \operatorname{Max} u_{i}\left(q_{i}, p_{-i}\right) .
$$

(iii) Update Phase. As illustrated from the MIA and DIA algorithms, nodes selected their power range in each iteration 
and determined their neighborhoods. Based on the detection of its neighbors, the node updates the overall topology. Each node changes its transmission range to a certain connected topology state. Additionally, other nodes are informed of this change by some optimized broadcast algorithm.

In DIA algorithm, each node minimizes its transmission power level since this change results in maximizing its utility; otherwise, the player changes transmission power to its previous power level. Additionally, the researcher used potential game in TC game for globally energy efficient. The MIA converges to topologies that ensure the connectivity establishment. However, the MIA is not energy efficient. On the other hand, the DIA algorithm guarantees convergence to min-max energy efficiency and preserves the network connectivity. Additionally, to guarantee the convergence to $\mathrm{NE}$, the nodes employ the best response algorithm to choose an adequate transmission power level. However, in DIA and MIA, nodes do not consider the residual energy and it may result in draining more energy.

Authors in [36] introduced a local minimum spanning tree (LMST) based algorithm for topology control. LMST is a localized algorithm to build minimum spanning tree based connected topology. Moreover, the LMST uses onehop neighbor's information of nodes. Each node has an identification ID. In the LMST algorithm, each node from the information of one-hop calculates MST individually. The researcher constructed LMST with the following two phases.

(i) Initially, each node sends a "hello message" using the maximal power level $d_{\max }$ as the undirected graph $G=(V, E)$, where $V$ is set of nodes and $E$ is set of link, $E=\left\{(u, v), d_{(u, v)}<\right.$ $d_{\max }, u, v \in V$. Hello message as visible neighborhood contains its ID that helps node to construct its local graph.

(ii) In second phases, after obtaining visible neighborhood, each node $u$ builds its local MST $T_{u}=\left(V\left(T_{u}\right), E\left(T_{u}\right)\right)$ of $G_{u}$ from its local graph. Each node uses "Bellman-Ford shortest" algorithm individually to obtain its local MST. Each link has a different weight; for example, edges $\left(u_{1}, v_{1}\right)$ and $\left(u_{2}, v_{2}\right)$ are different. The locally computed MST can preserve the network connectivity. To simply connectivity, authors in [36] defined neighborhood relation and neighborhood set. Node $v$ is a neighborhood of node $u$ 's, denoted by $u \rightarrow v$, if and only if $(u, v) \in E\left(T_{u}\right)$. Moreover, $u \leftrightarrow v$, if and only if $u \rightarrow v$ and $v \rightarrow u$. In addition, that is, node $v$ is a neighborhood of node $u$ 's if and only if the node $v$ is on the node $u$ 's LMST, $T(u)$ and is one-hop away from node $u$ as shown in Figure 6.

In addition, the proposed algorithm in [36] considered the pay-off of a node for adding a tree as a function of its transmission power. More accurately, every node is only concerned in minimizing its transmission level while being connected to the network. The one-hop neighborhood of each node is determined trivially based on its transmission power level. However, it is not an energy efficient solution for the large-scale network. Moreover, the node energy usage distribution in LMST is unbalance. Consequently, those nodes with high consumption rates may leading to the

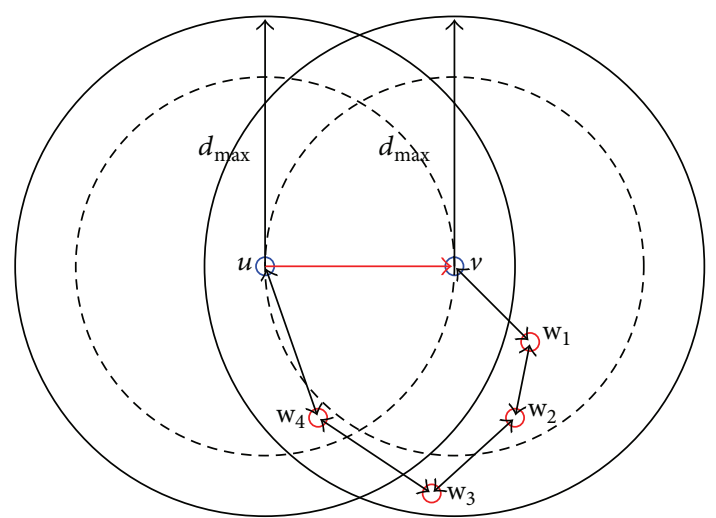

FIgure 6: The LMST.

network operational lifetime fault prematurely. Therefore, the battery usage of each node should be balanced.

In [41], authors introduced an algorithm to optimize the traditional TC scheme. In such algorithm, each node repetitively maximizes its power level. This algorithm starts from a symmetric, connected network, assumed to be the output of conventional of TC algorithms. Accordingly, more reliable TC algorithms were investigated in [54], such as fault-tolerant local and local tree based reliable topology (LTRT). Such algorithms can preserve $k$-link connectivity; that is, topology cannot be disconnected if the numbers of disconnected edges are less than $k$ and are referred to as $k$ link connected algorithm.

\subsubsection{Game Theory Based Network Capacity Topology Control} Algorithms. In [35], authors proposed multi-power topology control (MTC) game, where each node is capable to employ multichannel communications to minimize their energy consumption. Indeed, nodes do not adjust their transmission range for themselves, since it is assumed to have the capability of sending data at multiple power ranges simultaneously. However, the MTC algorithms are equipped with multiradio.

Authors in [21] proposed neighbor selection game (NS) with complete information of network. In NS algorithm, each node is interested to selfishly choose its neighbors such that their energy usage is reduced and the network capacity is improved. The NS algorithm considers the benefit of connectivity and energy cost of a node for connecting to a network. Each node is only trying to minimize its transmission range while being joined to other nodes as shown in Figure 7. The authors believed that the rate of energy consumed in a wireless network depends not only on transmission power but also on the amount of packets it forwards. Finally, in [21], authors introduced two distributed algorithms (global versus local) for obtaining a TC in a network in the presence of selfish nodes. In the global algorithm, each player knows the complete information about the network connectivity. While in the local model, each node gathers neighbor information within a limited hop. Then generalize the problem to the case where the transmission powers are the unknown variables and should be determined jointly with the neighbor sets. In addition, the authors consider the topology control and 


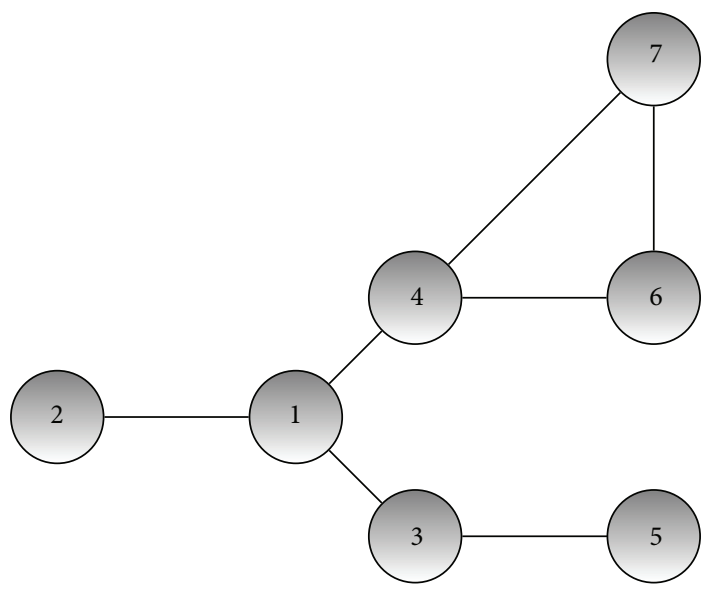

FIGURE 7: A sample NE topology in which no node benefits from removing any of the links.

neighbor selection as a joint process of joint neighbor Selection (JNS) algorithm in which both the transmission power and the neighbor set of nodes are unknown. The power list is not given as input to the problem; instead, nodes work on an initial topology to determine their transmission power and neighbor list together. In the JNS algorithms, players start with the max graph and in each repetition, one node takes turn and plays its best response and assumes that nodes have global knowledge about the connectivity of the whole network. Furthermore, the joint best response algorithm works similarly to the local method except that each node chooses the lightest link between itself and every $k$-hop strong component. The results of their simulations show that the global method yields about $20 \%$ higher total energy consumption than the approximated (stable) solution. However, refer to simulation results and the JNS algorithm needs fully global information to work properly. Based on the result, the local method can reduce this problem by more than $10 \%$.

4.2.4. Game Theory Based Energy Balance Topology Control Algorithms. Authors in [15] introduced virtual game-based energy balanced topology control algorithm (VGEB) with incomplete information. VGEB algorithm considers balancing energy consumption which can drain nodes energy. In the virtual game (VG), each node exchanges its complete information only once within neighbor nodes. Furthermore, in VGEB algorithm, first, each node will select some neighbor nodes with more remaining energy as their other neighbors to mitigate the energy usage of nodes with less remaining energy as shown in Figure 8. Additionally, in VGEB algorithm, each node $i$ constructs the VGEB $\Gamma^{i}=\left\langle N^{(i)}, P^{(i)}, u^{(i)}\right\rangle$ in which each node only makes a decision based on its power level. Following three phases describe the VGEB algorithm.

(i) Information Collection Phases. Each node $i$ sets its transmission power $p^{\max }$ and propagates request message for its neighborhood in $p^{\max }$. Since the node $i$ receives ACK from each responding neighborhood $j$, node $i$ contains neighborhood $j$ 's ID, transmission power level to node $j \mathrm{~s}, p_{i j}$, and residual energy into the order list. Based on the collected neighbor lists, each node $i$ has each node $k$ 's $(k \in N(i))$ power action set $P_{k}^{(i)}=\left\{p_{k 1}^{(i)}, p_{k 2}^{(i)}, \ldots, p_{k m_{k}}^{(i)}\right\}$, where $k$ is a symbol in $N(i)$.

(ii) Virtual Game Phases. In this phase, each node has discretized the action vector. The action set of node $k \in N(i)$ is defined as

$$
P_{k}^{(i)}=\left\{p_{k}^{\max }=p_{k 1}^{(i)}, p_{k 2}^{(i)}, \ldots, p_{k m_{k}}^{(i)}=p_{k}^{\max }\right\},
$$

where $k$ selects transmission level, one power levels less than the current level if the selected level gives higher utility than its certain power level. Otherwise, the node revers to the power range it is currently used. Given the transmission power level of all other nodes in $N^{i}$,

$$
p_{k}^{i}=\arg \max _{q_{k}^{i} \in p_{k h}^{i}, p_{k m}^{i}} u_{k}^{i}\left(q_{-k}^{i}, q_{k}^{i}\right),
$$

where $u_{k}^{i}\left(q_{-k}^{i}, q_{k}^{i}\right)$ is utility function which is outcome of each transmission power level. Additionally, a utility function obtains the trade-off and sets the power level action to a benefit for each node, based on the following equation (13):

$$
\begin{aligned}
& u_{k}^{i}\left(q_{-k}^{i}, q_{k}^{i}\right) \\
& \quad=f_{k}^{i}\left(q_{-k}^{i}, q_{k}^{i}\right)\left(\frac{\alpha p_{k}^{\max }}{E_{r k}}+\beta \bar{E}_{k}\left(p_{k}^{i}\right)\right)-\left(\frac{\alpha p_{k}^{i}}{E_{r k}}\right),
\end{aligned}
$$

where $\alpha$ and $\beta$ are nondecreasing value. $f_{k}^{i}\left(q_{-k}^{i}, q_{k}^{i}\right)=1$ if sensor node $k$ is able to connect to its neighbors, else $f_{k}^{i}\left(q_{-k}^{i}, q_{k}^{i}\right)=0$. Additionally, $E_{k}\left(p_{k}^{i}\right)$ is the average residual energy of a node $j$ 's, $j$ is the node in which node $k$ is able to be connected by one-hop distance with $p_{k}^{i}, E_{0}(j)$ and $E_{r}(j)$ are the initial energy allocated and the residual energy, respectively.

(iii) Maintenance Phase. As the operational time of the network goes by, energy usage of the nodes becomes unbalanced. It is possible for each node $i$ to figure out its own residual energy. Furthermore, if its residual energy is less than a specified level (e.g., a level is $1 / 5$ of its allocated battery), then node $i$ executes the VG algorithm $\Gamma^{(i)}\left(p^{\max } \rightarrow p^{(i)}\right)$ to readjust its transmission power level.

VGEB greatly minimizes the energy exhausting in the information exchange and considers the energy balance. However, if a topology only considers energy balancing without considering pursuing energy efficiency, node's lifetime may fail prematurely.

Traditional TC algorithms such as DRNG, DLSS [55], and STC [34] start the TC execution with each node's maximal transmission level to detect all of its neighbors. Local neighbor and transmission power range information is exchanged between nodes and their neighbors. Without further communication between nodes, the minimum power level of each node is calculated at each node. However, based on DRNG, DLSS and STC algorithms nodes do not have 


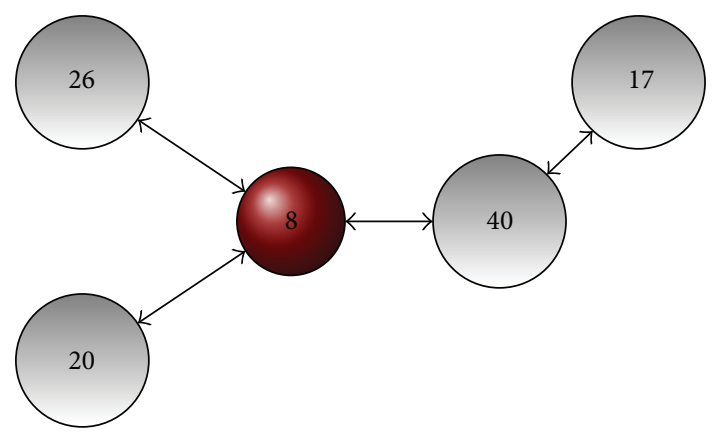

(a)

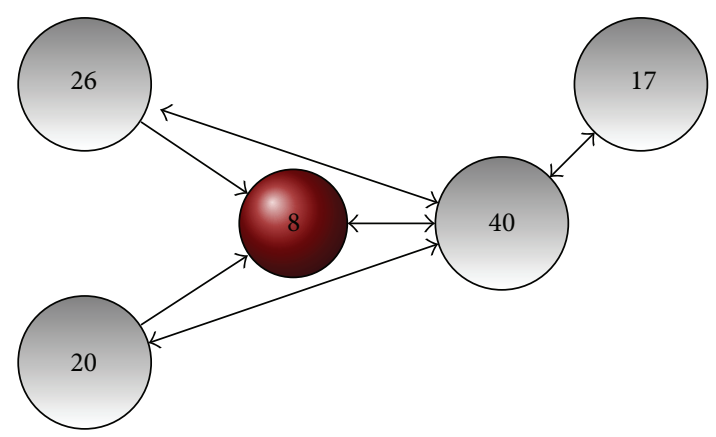

(b)

FIGURE 8: (a) The topology without considering remain energy (b) and the topology considering remaining energy.

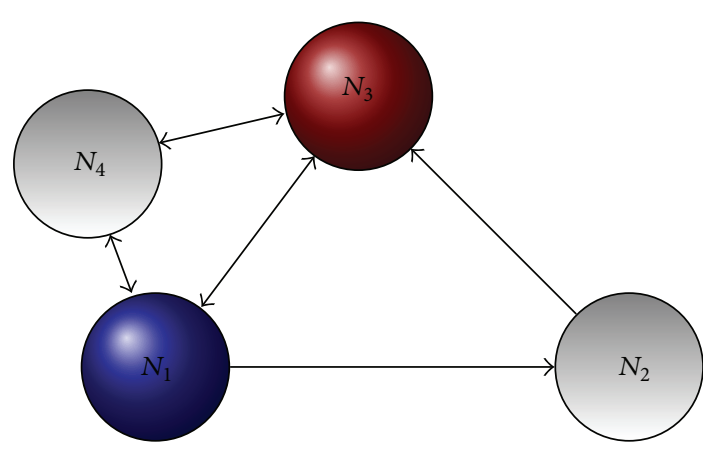

(a)

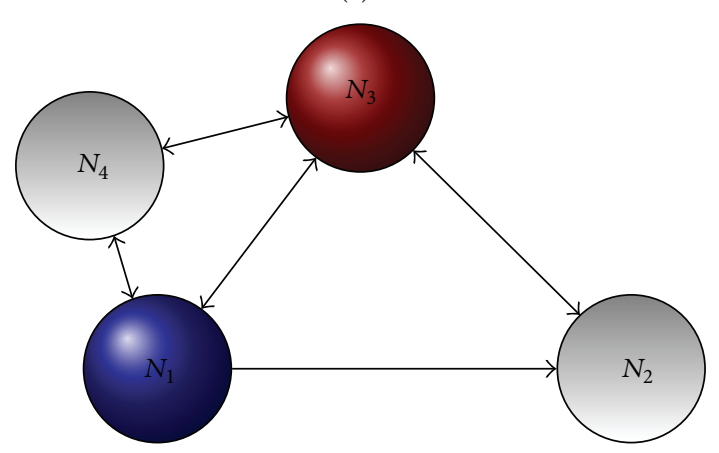

(c)

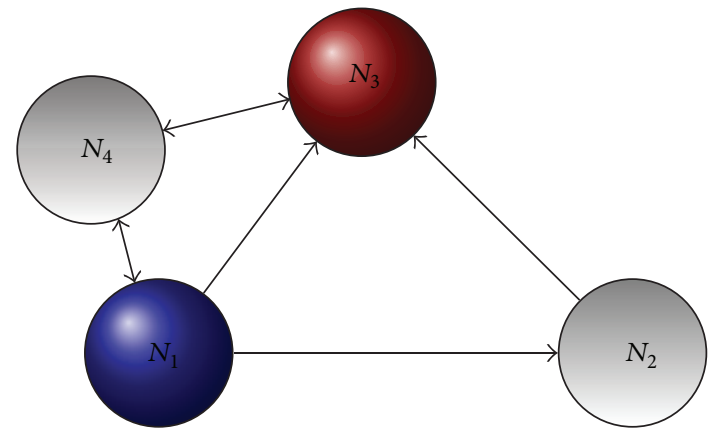

(b)

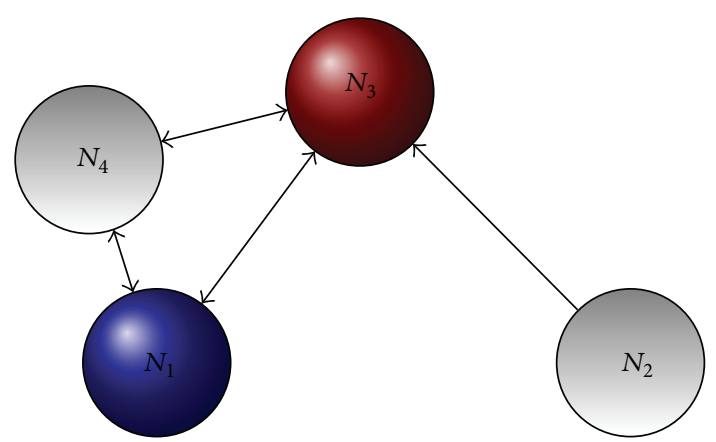

(d)

FIgURE 9: Transmission power selection in the CTCA algorithm. (a) Case 1: Node $N_{3}$ 's, where node $N_{1}$ is not able of minimizing its power level without being unconnected. (b) Case 2: Node $N_{3}$ minimizes its power level to its optimal power, but $N_{1}$ 's lifetime cannot be increased. (c) Case 3: Node $N_{3}$ maximizes its power level to $p\left(N_{3} ; N_{2}\right)$. Now, node $N_{1}$ is capable to minimize its power without being unconnected. (d) Case 4: Node $N_{1}$ modifies its certain power level to its potential power and improves lifetime of the network.

permission to cooperate with neighbor nodes to improve the network functional lifetime.

The approach in [42] proposed cooperative topology control with Adaptation (CTCA) algorithm. CTCA algorithm is adaptive and allows cooperation among nodes to increase the network operational lifetime. The CTCA consists of two main phases.

(i) Neighbor List Phases. Each node sets its power at the maximum transmission level $p_{\max }$ and makes neighbor list of a node $N_{i}$, that is, equal to $R_{i}(0)=\left\{N_{j} p\left(N_{i}, N_{j} \leq p_{\max }\right)\right\}$. Determine the action set for node $N_{i}$ as $A_{i}=\left\{p_{i}^{1}, p_{i}^{2}, \ldots, p_{i}^{n}\right\}$, where, for node $N_{j} \in R_{i}(0)$, there exists a power range $p_{k}^{i} \in A_{i}$. Moreover, $p_{k}$ is the lower power level required for $N_{i}$ to connect $N_{j}$. The proposed CTCA algorithm assumed that $p_{i}^{1} \leq p_{i}^{2} \leq p_{i}^{n} \leq p_{\max }$. The CTCA algorithm uses DLSS algorithm [55] for determining $p_{i}$ for transmission power level adjustment. Moreover, each node propagates message to its neighbor for current residual energy by mark "energy info shared" $W_{i}(t)$ and modifies $W_{j}$ for $N_{j} \in I_{i}$.

(ii) Neighbor Assisted Power Adjusts Phases (NAPA). Each node tries to improve its neighbor's operational life, refereed by $N_{m_{(i)}}(t)$ the node in $O_{i}(t)=I_{i}(t) \cup N_{i}$ with the lower 
functional life as shown in Figure 9. Moreover, its optimal transmission level at interval $t$ is set as $Y_{m_{(i)}}\left(P_{m}(t)\right)$, and its functional life at $t$ is set as $Z_{m_{(i)}}\left(P_{m}(t)\right)$. In addition, assumption $a_{i} \in A_{i}$ refers to the current transmission power for node $N_{i}$ in time $t$. CTCA algorithm determines the utility $u_{i}$ for node $N_{i}$ with power level $a_{i}$ as

$$
\begin{aligned}
u_{i}\left(a_{i}, t\right)= & c_{i}\left(a_{i}, t\right) V_{i}\left(a_{i}, t\right) l_{i}\left(a_{i}, t\right) \\
& +c_{i}\left(a_{i}, t\right) \min \left(\min _{N_{j} \in I_{i}(t)} V_{i}\left(a_{i}, t\right), Z_{j}\left(P_{j}(t), t\right)\right),
\end{aligned}
$$

where $c_{i}\left(a_{i}, t\right)$ denotes 0,1 . If $c_{i}\left(a_{i}, t\right)=1$ node, $N_{i}$ can connect to a node $N_{j}$ with power range $a_{i}$; otherwise, $c_{i}\left(a_{i}, t\right)=0$. $c_{i}\left(a_{i}, t\right)$ is used to show the node $N_{i}$ operational lifetime by transmission power $a_{i}$. If $N_{m_{(i)(t)}}$ 's current power level can be minimized by $N_{i}$ transmitting at power range $a_{i}$ and $N_{m}(i)$ functional life in this case is greater than the neighbor's lifetime. Additionally, $N_{m}(i)$ 's operational life is not considered, and therefore $l_{i}\left(a_{i}, t\right)=0$. However, if transmitting at the transmission level $a_{i}$ may not help increasing $N_{m_{(i)(t)}}$ 's operational life, its operational life results in lower than the neighbor's operational lifetime in the connected network. In addition, node $N_{i}$ should increase its operational life; that is, $l_{i}\left(a_{i}, t\right)=1$.

If the CTCA algorithm meets all of the conditions illustrated above, then node $N_{i}$ will be selected to maximize its transmission level in order to help increasing its neighbor's functional life. Furthermore, the author had proven the existence of a NE for the game theory and provided a algorithm which gains a NE. The simulation results of the CTCA algorithm show that the algorithm is able to increase the functional life and balance energy consumption. However, the proposed algorithm is not an energy efficient solution, which consume more energy with using high power level. Consequently, those nodes with high consumption rates are leading to the network operational lifetime over prematurely. Therefore, topology control should consider energy efficiency and energy balancing together.

\section{Discussion}

Topology control of wireless networks is unstructured to fluctuations. To tackle such unstable characteristics, a TC mechanism must overcome the variability in typologies. The fundamental aim of TC is that, instead of using the maximum transmission level, each node collaboratively adjusted its power level and constructed efficient topology, with the objective of improving the network functional lifetime. Table 1 shows the main characteristics of the existing TC algorithms. It identifies the algorithm of each algorithm and clarifies the class of these algorithms. The objectives of each algorithm are also included. Most current TC algorithms for energy conservation have inconsecutive assumptions.

Obviously, it is the connectivity at the edge that qualifies end-to-end connectivity. The well-known link connectivity is the protocol model or disk model. Connectivity problem is considered to minimize one of the power adjustments.
The comprehensive feature of deterministic TC algorithms can be stated as an optimization problem. Additionally, the objective, mainly, is to specify a power adjustment, such that $p_{i}^{\max }$ to $p(i)$ is decreased, while preserving connectivity. Moreover, the objective could be desirable with following properties: $k$-connectivity, node degree, and spanner.

There are numerous topology control designed for WSNs and ad hoc networks such as homogeneous transmission power control and heterogeneous transmission power control. The homogeneous transmission power control can be characterized in two schemes, centralized and distributed topology control scheme. In centralized topology control, nodes need authority to control. In distributed topology control, each node act selfishly to save its limited energy in critical environment by using its neighbor information. Such information can be achieved by exchanging information between the nodes. On the other hand, there are several challenges for topology control in wireless networks such as node deployment, network capacity, and energy consumption. However, energy consumption can be considered as the main important technical challenge for topology control in wireless networks. Obviously, energy efficiency topology control can be improved in two ways: minimizing the energy consumption by selecting optimum transmission power level or balancing energy consumption among the nodes. In all TC algorithm as mentioned in the literature, the only consideration for each node is to reduce its power level while preserving network connectivity. However, existing algorithms do not consider the fact that various nodes are in various positions in the topology, and some may end up with a high transmission power level. Therefore, this high power level will consume more energy and disrupt network. Also, many algorithms have been investigated to construct an efficient topology such that balanced energy consumption, for example, by considering residual energy of the node during transmission power selection.

Most algorithms assumed that nodes are cooperative to each other, since, in wireless networks, there is no method to qualify nodes. However, this assumption may not always hold. Each node may compete with its neighbor to conserve its own limited energy and consequently degrade the whole network efficiency. If the nodes select too low transmission power level, the constructed topology will be disconnected. It is more reasonable to assume that nodes act selfishly and this selfish behavior can be modeled as noncooperative games. Game theory is a fundamental tool to achieve an energy balance and energy efficient while preserving network connectivity in the present of selfish nodes. Moreover, in such models, nodes interact with other nodes to maximize their individual utility. Currently, the fundamental algorithm for noncooperative TC is based on adapting the power level of nodes, while the topology remains connected. However, some algorithms consider only energy balance and do not consider energy efficiency and reliable neighbor selection.

Though, there is a significant improvement in the theoretical study of energy efficiency topology control in wireless networks. There is some evidence to confirm benefits of TC on improving network lifetime. In fact, distinguishing 
optimal power selection and reliable neighbor selection that can forward packets through the network at rates close to capacity with minimum energy consumption is still an open issue.

\section{Conclusions}

In this paper, topology control aims of the existing algorithms are characterized into three main types: energy efficiency, network capacity, and energy balancing. The most famous and recent topology control algorithms based on their features are reviewed and compared in each goals category. According to this overview, most of the proposed algorithms consider minimizing the transmission power levels while preserving network connectivity in wireless network (DIA, MIA, and OTTC). Furthermore, some of them consider balanced energy consumption of nodes (VGEB, CTCA, and ECR) and a number of them aim to improve network capacity (NS, RETC). However, by increasing the application of wireless networks, the functions of wireless nodes will be so highlighted and they always seek to achieve conflicting objectives such as in the pursuit of energy efficiency and network connectivity. Therefore, scalable solutions which can perform topology control by considering multiobjective QoS requirements and high spatial reuse are greatly required for wireless networks.

\section{References}

[1] I. F. Akyildiz, W. Su, Y. Sankarasubramaniam, and E. Cayirci, "A survey on sensor networks," IEEE Communications Magazine, vol. 40, no. 8, pp. 102-105, 2002.

[2] P. Santi and J. Simon, "Silence is golden with high probability: maintaining a connected backbone in wireless sensor networks," in Wireless Sensor Networks, pp. 106-121, 2004.

[3] P. Santi, "Topology control in wireless ad hoc and sensor networks," ACM Computing Surveys, vol. 37, no. 2, pp. 164-194, 2005.

[4] S. E. Calvano, W. Xiao, D. R. Richards et al., "A network-based analysis of systemic inflammation in humans," Nature, vol. 437, no. 7061, pp. 1032-1037, 2005.

[5] N. Mladineo and S. Knezic, "Optimisation of forest fire sensor network using gis technology," in Proceedings of the 22nd International Conference on Information Technology Interfaces (ITI '02), pp. 391-396, 2000.

[6] L. Ritchie, S. Deval, M. Reisslein, and A. W. Richa, "Evaluation of physical carrier sense based spanner construction and maintenance as well as broadcast and convergecast in ad hoc networks," Ad Hoc Networks, vol. 7, no. 7, pp. 1347-1369, 2009.

[7] R. S. Komali, A. B. MacKenzie, and R. P. Gilles, "Effect of selfish node behavior on efficient topology design," IEEE Transactions on Mobile Computing, vol. 7, no. 9, pp. 1057-1070, 2008.

[8] T. M. Chiwewe and G. P. Hancke, "A distributed topology control technique for low interference and energy efficiency in wireless sensor networks," IEEE Transactions on Industrial Informatics, vol. 8, no. 1, pp. 11-19, 2012.

[9] R. S. Komali and A. B. MacKenzie, "Distributed topology control in ad-hoc networks: a game theoretic perspective," in Proceedings of the 3rd IEEE Consumer Communications and Networking Conference (CCNC '06), pp. 563-568, January 2006.
[10] Z. Mi and Y. Yang, "Topology control and coverage enhancement of dynamic networks based on the controllable movement of mobile agents," in Proceedings of the IEEE International Conference on Communications (ICC '11), pp. 1-5, June 2011.

[11] S.-C. Wang, D. S. L. Wei, and S.-Y. Kuo, "An SPT-based topology control algorithm for wireless ad hoc networks," Computer Communications, vol. 29, no. 16, pp. 3092-3103, 2006.

[12] Y. Wang, "Topology control for wireless sensor networks," in Wireless Sensor Networks and Applications, pp. 113-147, 2008.

[13] C. Song, M. Liu, J. Cao, Y. Zheng, H. Gong, and G. Chen, "Maximizing network lifetime based on transmission range adjustment in wireless sensor networks," Computer Communications, vol. 32, no. 11, pp. 1316-1325, 2009.

[14] X. Wu, G. Chen, and S. K. Das, "Avoiding energy holes in wireless sensor networks with nonuniform node distribution," IEEE Transactions on Parallel and Distributed Systems, vol. 19, no. 5, pp. 710-720, 2008.

[15] X.-C. Hao, Y.-X. Zhang, N. Jia, and B. Liu, "Virtual game-based energy balanced topology control algorithm for wireless sensor networks," Wireless Personal Communications, pp. 1-20, 2012.

[16] C. Schurgers, V. Tsiatsis, and M. B. Srivastava, "Stem: topology management for energy efficient sensor networks," in IEEE Aerospace Conference Proceedings, vol. 3, pp. 1099-1108, 2002.

[17] S. Zarifzadeh, A. Nayyeri, and N. Yazdani, "Efficient construction of network topology to conserve energy in wireless ad hoc networks," Computer Communications, vol. 31, no. 1, pp. 160173, 2008.

[18] S. Rizvi, H. K. Qureshi, S. Ali Khayam, V. Rakocevic, and M. Rajarajan, "A1: an energy efficient topology control algorithm for connected area coverage in wireless sensor networks," Journal of Network and Computer Applications, vol. 35, no. 2, pp. 597-605, 2012.

[19] S. Eidenbenz, V. S. A. Kumar, and S. Zust, "Equilibria in topology control games for ad hoc networks," Mobile Networks and Applications, vol. 11, no. 2, pp. 143-159, 2006.

[20] R. S. Komali, R. W. Thomas, L. A. Dasilva, and A. B. MacKenzie, "The price of ignorance: distributed topology control in cognitive networks," IEEE Transactions on Wireless Communications, vol. 9, no. 4, pp. 1434-1445, 2010.

[21] S. Zarifzadeh and N. Yazdani, "Neighbor selection game in wireless ad hoc networks," Wireless Personal Communications-, vol. 70, no. 2, pp. 617-640, 2013.

[22] M. J. Osborne, An Introduction to Game Theory, vol. 3, Oxford University Press, New York, NY, USA, 2004.

[23] J. Ratliff, "A folk theorem sampler. Lecture notes," 2010, http:// www.virtualperfection.com/gametheory/5.3.FolkTheoremsampler.1.0.pdf.

[24] G. Arslan and J. S. Shamma, "Distributed convergence to nash equilibria with local utility measurements," in Proceedings of the 43rd IEEE Conference on Decision and Control (CDC '04), pp. 1538-1543, December 2004.

[25] J. R. Marden, G. Arslan, and J. S. Shamma, "Joint strategy fictitious play with inertia for potential games," IEEE Transactions on Automatic Control, vol. 54, no. 2, pp. 208-220, 2009.

[26] D. Monderer and L. S. Shapley, "Potential games," Games and Economic Behavior, vol. 14, no. 1, pp. 124-143, 1996.

[27] W. Poundstone and N. Metropolis, "Prisoner's dilemma: John von neumann, game theory, and the puzzle of the bomb," Physics Today, vol. 45, article 73, 1992. 
[28] J. Liu and B. Li, "Distributed topology control in wireless sensor networks with asymmetric links," in IEEE Global Telecommunications Conference (GLOBECOM '03), pp. 1257-1262, December 2003.

[29] H. Zhang and J. C. Hou, "Maintaining sensing coverage and connectivity in large sensor networks," Ad Hoc \& Sensor Wireless Networks, vol. 1, no. 1-2, pp. 89-124, 2005.

[30] X. Wang, G. Xing, Y. Zhang, C. Lu, R. Pless, and C. Gill, "Integrated coverage and connectivity configuration in wireless sensor networks," in Proceedings of the 1st International Conference on Embedded Networked Sensor Systems (SenSys '03), pp. 28-39, November 2003.

[31] P. Gupta and P. R. Kumar, "The capacity of wireless networks," IEEE Transactions on Information Theory, vol. 46, no. 2, pp. 388404, 2000.

[32] R. Ramanathan and R. Rosales-Hain, "Topology control of multihop wireless networks using transmit power adjustment," in Proceedings of a IEEE 19th Annual Joint Conference of the Computer and Communications Societies (INFOCOM '00), vol. 2, pp. 404-413, March 2000.

[33] M. Kadivar, M. E. Shiri, and M. Dehghan, "Distributed topology control algorithm based on one- and two-hop neighbors' information for ad hoc networks," Computer Communications, vol. 32, no. 2, pp. 368-375, 2009.

[34] H. Sethu and T. Gerety, "A new distributed topology control algorithm for wireless environments with non-uniform path loss and multipath propagation," Ad Hoc Networks, vol. 8, no. 3, pp. 280-294, 2010.

[35] S. Zarifzadeh, N. Yazdani, and A. Nayyeri, "Energy-efficient topology control in wireless ad hoc networks with selfish nodes," Computer Networks, vol. 56, no. 2, pp. 902-914, 2012.

[36] N. Li, J. C. Hou, and L. Sha, "Design and analysis of an MST-based topology control algorithm," IEEE Transactions on Wireless Communications, vol. 4, no. 3, pp. 1195-1206, 2005.

[37] R. Wattenhofer and A. Zollinger, "XTC: a practical topology control algorithm for ad-hoc networks," in Proceedings of the 18th International Parallel and Distributed Processing Symposium (IPDPS '04), pp. 2969-2976, April 2004.

[38] P. Santi and D. M. Blough, "The critical transmitting range for connectivity in sparse wireless ad hoc networks," IEEE Transactions on Mobile Computing, vol. 2, no. 1, pp. 25-39, 2003.

[39] J. Yu, H. Roh, W. Lee, S. Pack, and D. -Z. Du, "Topology control in cooperative wireless ad-hoc networks," IEEE Journal on Selected Areas in Communications, vol. 30, no. 9, pp. 17711779, 2012.

[40] J. Li and P. Mohapatra, "Analytical modeling and mitigation techniques for the energy hole problem in sensor networks," Pervasive and Mobile Computing, vol. 3, no. 3, pp. 233-254, 2007.

[41] M. Cardei, J. Wu, and S. Yang, "Topology control in ad hoc wireless networks using cooperative communication," IEEE Transactions on Mobile Computing, vol. 5, no. 6, pp. 711-724, 2006.

[42] X. Chu and H. Sethu, "Cooperative topology control with adaptation for improved lifetime in wireless ad hoc networks," in Proceedings of the 28th IEEE International Conference on Computer Communications (INFOCOM '12), pp. 262-270, 2012.

[43] S. Olariu and I. Stojmenović, "Design guidelines for maximizing lifetime and avoiding energy holes in sensor networks with uniform distribution and uniform reporting," in Proceedings of the 25th IEEE International Conference on Computer Communications (INFOCOM '06), vol. 6, pp. 1-12, April 2006.
[44] R. Sun, J. Yuan, I. You, X. Shan, and Y. Ren, "Energy-aware weighted graph based dynamic topology control algorithm," Simulation Modelling Practice and Theory, vol. 19, no. 8, pp. 1773-1781, 2011.

[45] C.-Y. Lee, L.-C. Shiu, F.-T. Lin, and C.-S. Yang, "Distributed topology control algorithm on broadcasting in wireless sensor network," Journal of Network and Computer Applications, vol. 36, pp. 1186-1195, 2013.

[46] V. Srivastava, J. Neel, A. B. MacKenzie et al., "Using game theory to analyze wireless ad hoc networks," IEEE Communications Surveys and Tutorials, vol. 7, no. 4, pp. 46-56, 2005.

[47] M. D. Penrose, "The longest edge of the random minimal spanning tree," The Annals of Applied Probability, vol. 7, no. 2, pp. 340-361, 1997.

[48] L. M. Kirousis, E. Kranakis, D. Krizanc, and A. Pelc, "Power consumption in packet radio networks," Theoretical Computer Science, vol. 243, no. 1-2, pp. 289-305, 2000.

[49] A. E. F. Clementi, P. Penna, and R. Silvestri, "Hardness results for the power range assignment problem in packet radio networks," in Algorithms and Techniques of Randomization, Approximation, and Combinatorial Optimization, pp. 197-208, 1999.

[50] J. Lian, K. Naik, and G. B. Agnew, "Data capacity improvement of wireless sensor networks using non-uniform sensor distribution," International Journal of Distributed Sensor Networks, vol. 2, no. 2, pp. 121-145, 2006.

[51] A. Jarry, P. Leone, O. Powell, and J. Rolim, "An optimal data propagation algorithm for maximizing the lifespan of sensor networks," in Distributed Computing in Sensor Systems, pp. 405421, 2006.

[52] S. Yuen and B. Li, "Strategyproof mechanisms towards dynamic topology formation in autonomous networks," Mobile Networks and Applications, vol. 10, no. 6, pp. 961-970, 2005.

[53] P. Santi, S. Eidenbenz, and G. Resta, "A framework for incentive compatible topology control in non-cooperative wireless multihop networks," in Proceedings of the Workshop on Dependability Issues in Wireless Ad Hoc Networks and Sensor Networks (DIWANS '06), pp. 9-18, September 2006.

[54] K. Miyao, H. Nakayama, N. Ansari, and N. Kato, "LTRT: an efficient and reliable topology control algorithm for ad-hoc networks," IEEE Transactions on Wireless Communications, vol. 8, no. 12, pp. 6050-6058, 2009.

[55] N. Li and J. C. Hou, "Localized topology control algorithms for heterogeneous wireless networks," IEEE/ACM Transactions on Networking, vol. 13, no. 6, pp. 1313-1324, 2005. 


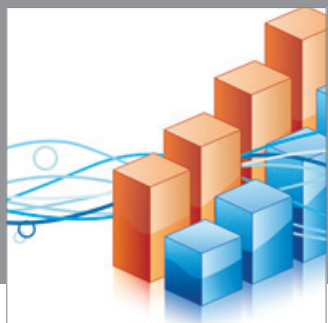

Advances in

Operations Research

mansans

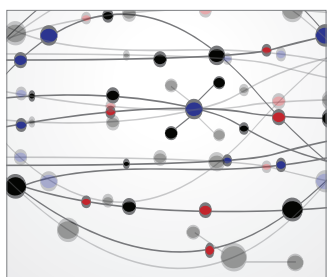

The Scientific World Journal
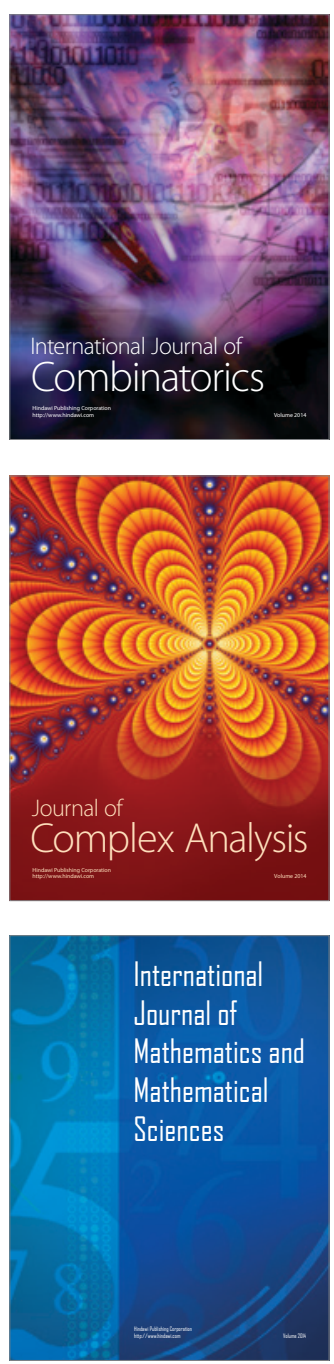
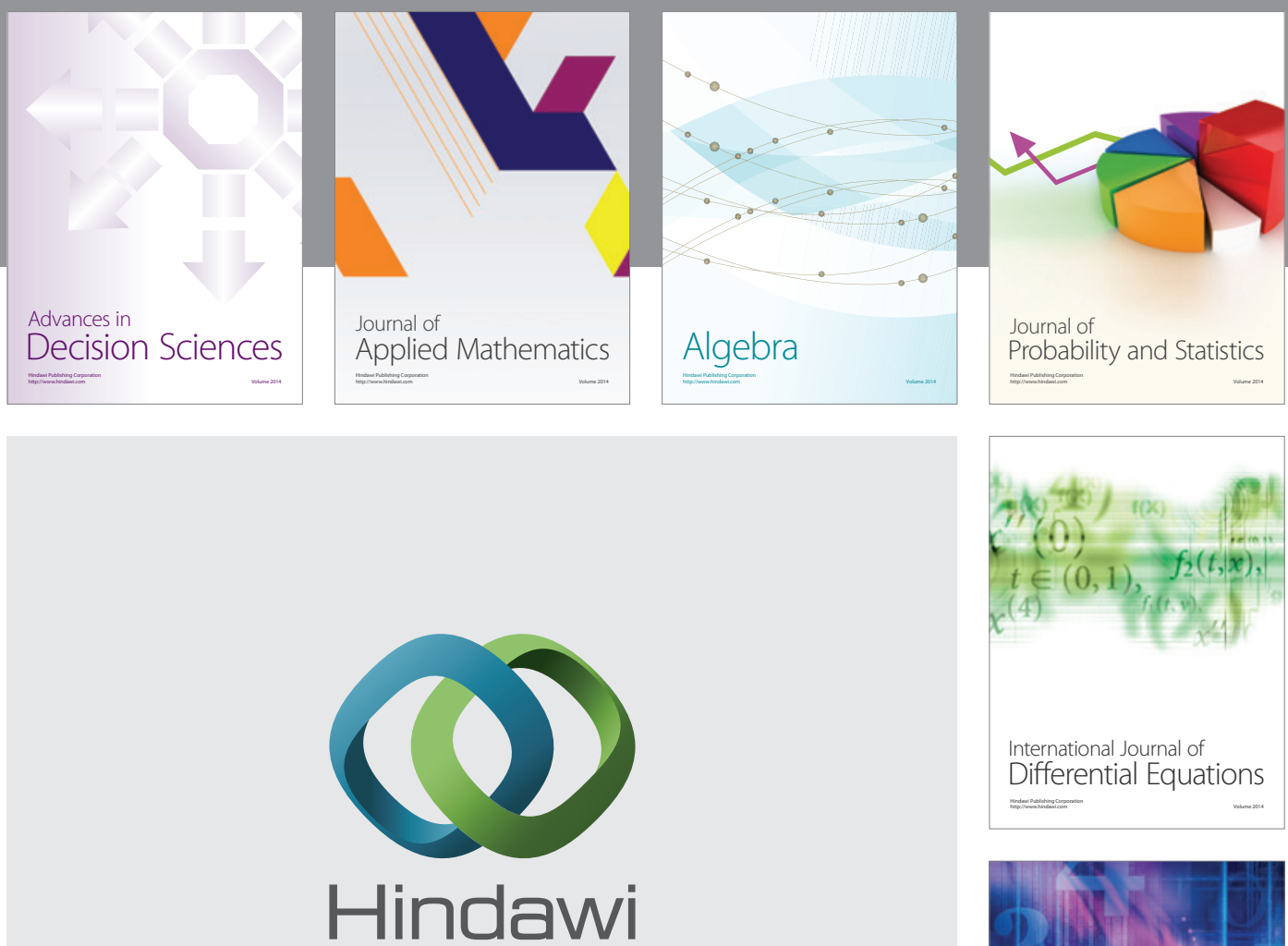

Submit your manuscripts at http://www.hindawi.com
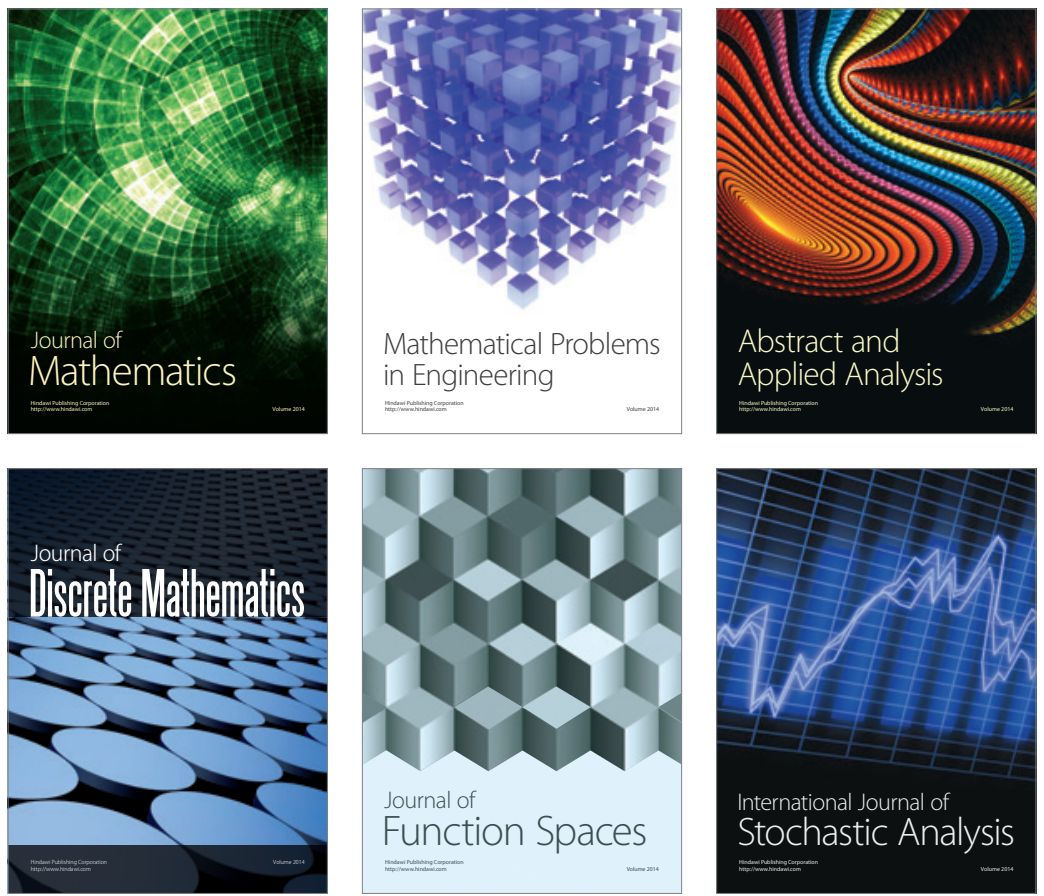

Journal of

Function Spaces

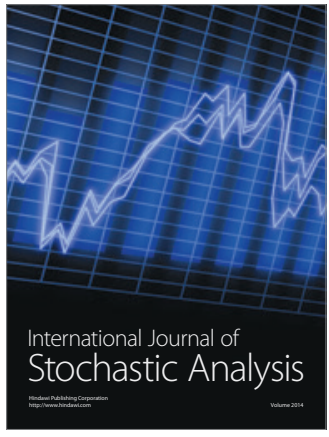

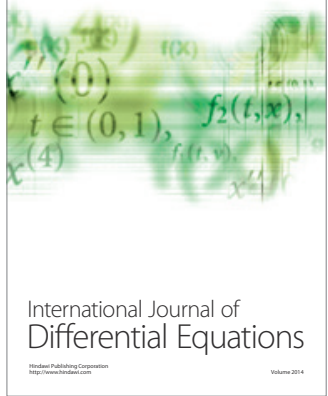
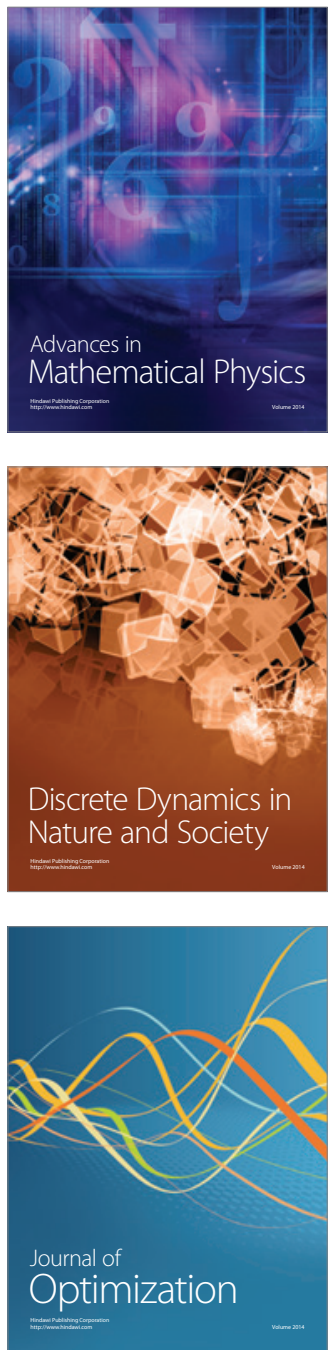